\title{
Philosophiques
}

\section{Sémiotique et abstraction : de Locke à Condillac}

\section{François Duchesneau}

Volume 3, numéro 2, octobre 1976

URI : https://id.erudit.org/iderudit/203051ar

DOI : https://doi.org/10.7202/203051ar

Aller au sommaire du numéro

Éditeur(s)

Société de philosophie du Québec

ISSN

0316-2923 (imprimé)

1492-1391 (numérique)

Découvrir la revue

Citer cet article

Duchesneau, F. (1976). Sémiotique et abstraction : de Locke à Condillac.

Philosophiques, 3(2), 147-166. https://doi.org/10.7202/203051ar d'utilisation que vous pouvez consulter en ligne.

https://apropos.erudit.org/fr/usagers/politique-dutilisation/ 


\title{
SÉMIOTIQUE ET ABSTRACTION : DE LOCKE À CONDILLAC
}

\author{
par François Duchesneau
}

Pour les philosophes des Lumières, l'Essay concerning Human Understanding de Locke reste le maître livre de la nouvelle métaphysique, en dépit des critiques de Berkeley, qui visent à démanteler certains éléments stratégiques de la philosophie lockienne. Berkeley s'était attaqué, en premier lieu, à la doctrine des idées abstraites et sa critique lui fournissait les éléments de base de l'immatérialisme. Condillac et ses successeurs veulent défendre la cause de la science, source des progrès de l'esprit humain, contre le scepticisme inhérent à la position de Berkeley. Il s'agit pour eux de repenser les critiques que Berkeley adressait à la philosophie du maître de sa jeunesse. Or la critique des idées abstraites leur semble tout à fait pertinente, et sans doute irréfutable. Comment surmonter la difficulté ? La solution pouvait-elle se trouver dans une modification de la perspective philosophique? Peut-être s'agissait-il de passer d'une philosophie centrée sur l'analyse critique de l'entendement humain à une philosophie de la nature humaine, en achevant ainsi le projet de la critique philosophique? C'est cet itinéraire de pensée dont nous allons tenter de retracer les grandes lignes.

\section{I - L'ANALYSE PROBLÉMATIQUE DE L'ENTENDEMENT CHEZ BERKELEY}

Berkeley, voulant dénoncer les faux principes qui entraînent la spéculation philosophique vers le scepticisme, s'en prend à l'opinion " that the mind has a power of framing abstract ideas or notions of things $"^{1}$. Il n'y a pas, affirme-t-il, de représentations

1. Berkeley, A Treatise concerning the Principles of Human Knowledge (1710), Introd., 6. Cet ouvrage est incontestablement le plus significatif de Berkeley en ce qui concerne le problème des idées abstraites. 
proprement générales, mais seulement des idées particulières : je puis, tout au plus, considérer à part des parties ou des qualités qui, bien qu'unies dans quelque objet, peuvent exister indépendamment de lui ${ }^{2}$. En conséquence, il formule des critiques à l'égard de la théorie des idées générales de Locke. Dans l'Essay, Locke situe en la possibilité de former des idées générales la distinction de la raison des hommes par rapport à celle des bêtes, laquelle est limitée à la consécution empirique des idées particulières, faute d'utiliser des signes pour représenter des idées générales ${ }^{3}$. Berkeley conteste que l'usage de termes généraux implique la formation d'idées générales : si le signe est général, c'est qu'il peut indifféremment suggérer à l'esprit l'une ou l'autre d'un grand nombre d'idées particulières. L'axiome " tout ce qui est étendu, est divisible " ne tient pas sa vérité de l'évidence d'une relation entre idées abstraites d'étendue et de divisibilité, mais il la tient de la capacité que j'ai d'appliquer cette règle à la série infinie des représentations particulières possibles de l'étendue, sans qu'elle soit réfutée par l'expérience. S'il n'y a pas d'idées générales abstraites, une idée particulière peut devenir générale dans la mesure où elle sert de signe pour représenter une classe d'idées particulières. La généralité ne serait donc pas issue de l'abstraction, mais d'une disposition de l'esprit à constituer des signes généraux, ou plutôt à attribuer à des signes une fonction représentative générale. Ainsi, dans le cas de la démonstration du théorème de géométrie suivant lequel la somme des angles intérieurs d'un triangle est égale à deux droits, l'idée que j'ai du triangle est la représentation d'une figure déterminée et unique, mais la démonstration vaut pour tous les triangles rectilignes, puisque, dans la démonstration, l'attention de l'esprit s'est détournée des caractéristiques particulières de la figure pour n'envisager que les propriétés qui permettent au triangle représenté d'être le type de tous les autres triangles rectilignes ${ }^{4}$. La double erreur de Locke, qui entache sa réforme de l'entendement humain, résulte de sa théorie de l'abstraction : elle vient de ce qu'il croyait " that the only immediate use of words was to signify ideas, and that the immediate signification of every general name was a determinate abstract idea "s. Comment l'illusion s'explique-t-elle?

\footnotetext{
2. Berkeley, Ibid., Introd., 10.

3. Locke, Essay concerning Human Understanding (1690), II, xi, 10-11.

4. Berkeley, Ibid., Introd., 16.

5. Berkeley, Ibid., Introd., 23.
} 
Berkeley l'explique par le principe méthodologique qui semble essentiel au travail scientifique : il faut donner aux termes un sens précis et déterminé ${ }^{6}$. Ce principe rend les philosophes enclins à penser qu'il y a quelque idée générale abstraite qui constitue la seule véritable signification d'un nom général. Or un nom général correspond à un grand nombre d'idées particulières qu'il peut indifféremment signifier. Et qu'on n'objecte pas qu'on peut donner une définition stable d'un nom, ce qui prouve qu'il signifie une idée abstraite : car autre chose est d'annexer la même définition au nom, autre chose de lui adjoindre la même idée ; il est inutile et impraticable de lier toujours la même idée au même mot'. S'il y a corrélation du mot et d'une idée, celle-ci, dans sa nature même, reste toujours particulière ; mais, et ce point est important, les mots ne correspondent même pas nécessairement à des représentations ou à des idées déterminées : car, même lorsque le langage a comme fonction de communiquer des idées, les noms signifiants ne sont pas toujours appelés à éveiller dans l'esprit les idées qu'ils sont censés représenter ; ils peuvent avoir un rôle symbolique analogue à celui des lettres en algèbre, qui indiquent un rapport de quantité à l'exclusion de toute quantité particulière. Par ailleurs, le langage n'a pas comme fin essentielle la communication des idées: "there are other ends, as the raising of some passion, the exciting to or deterring from an action, the putting the mind in some particular disposition $\aleph^{8}$. À cette fonction pratique, vitale, mais il vaudrait mieux dire naturelle suivant le qualificatif le plus approprié à la métaphysique du XVIIIe siècle, est subordonnée la fin de communication des idées, qui peut s'élider complètement, en particulier lorsque l'habitude intervient et rend le langage directement actif sur la sensibilité sans l'intermédiaire d'une représentation. À la règle de méthode qui stipule la détermination du sens des mots, Berkeley substitue la règle de séparation entre les idées et les mots : "I confine my thoughts to my own ideas divested of words $"$. Car les signes généraux - en particulier les mots

6. Locke, Ibid., III, xi, 8-9.

7. Berkeley, Ibid.. Introd., 18 ; mais Berkeley attribue ici à Locke une conception de la définition qui n'est pas la sienne. Bien qu'il soit réaliste en ce qui concerne les idées générales, Locke réduit l'usage des définitions à n'être que des définitions de mots : cf. Essay, III, iv, 6: "I think it is agreed, that a definition is nothing else but the showing the meaning of one word by several other not synonymous terms. "

8. Berkeley, Ihid., Introd., 20.

9. Berkeley, Ihid., Introd., 22. 
des différentes langues - ont une utilité pratique, mais n'ont pas d'usage spéculatif garanti par la possibilité qu'aurait l'esprit de former des idées abstraites. D'où la nécessité d'une science entièrement fondée sur l'expérience, qui ne serait rien d'autre que la perception commune débarrassée des préjugés philosophiques ${ }^{10}$.

Mais dans ces conditions, le problème est d'expliquer la science elle-même comme savoir rationnel : car d'où vient la nécessité qui lie des représentations particulières pour en faire une règle générale, voire universelle, capable d'étendre le savoir audelà de la perception actuelle et d'anticiper sur l'expérience à venir, comme c'est le cas lorsque le "géomètre " formule les lois de la mécanique céleste? Berkeley est obligé de considérer nos perceptions dans leur corrélation empirique comme un système de signes: "Those men who frame general rules from the phenomena and afterwards derive the phenomena from those rules seem to consider signs rather than causes ", ${ }^{11}$ ce qui s'explique par le fait qu'aucune réalité sensible ne peut proprement être dite cause, étant

10. Berkeley, Ibid., Introd., 24 : "In vain do we extend our view into the heavens and pry into the entrails of the earth, in vain do we consult the writings of learned men and trace the dark footsteps of antiquity, - we need only draw the curtain of words, to behold the fairest tree of knowledge, whose fruit is excellent and within the reach of our hand."

Il est toutefois évident que ce projet, lui-même, requiert une interprétation. Il semble que, pour Berkeley, la vérité de la science ne puisse avoir qu'une base empirique, puisqu'il s'agit d'une corrélation des phénomènes par l'usage de signes représentant les constantes de l'expérience sensible : ce qui rapproche la science humainement possible du statut de la perception commune. Quant à la prétention de fixer l'attention sur les idées, en se passant de toute médiation de signes (mots, en particulier), Berkeley la conçoit comme théoriquement fondée, mais, dans la pratique, il s'agit de rapporter l'usage des signes aux données mêmes de l'expérience sensible. Sur ce point, Berkeley semble retrouver un thème proprement lockien.

11. Berkeley, Ibid, 108. Il faut noter que, pour Locke, une science purement rationnelle, c'est-à-dire déductible a priori des phénomènes naturels, est inaccessible à l'homme. Et il insiste pour mettre en valeur le projet d'une science des phénomènes qui reposerait sur l'usage de la méthode historique, c'est-à-dire descriptive. $\grave{A}$ ce propos, J.W. Yolton, dans : Locke and the Compass of Human Understanding, Cambridge, C.U.P., 1970, a souligné l'ambition qu'avait Locke de soutenir l'entreprise scientifique de la Royal Society. Nos recherches personnelles nous ont amené à mettre en valeur cette forme de la science lockienne en rapport avec la méthode médicale. Cf. "La philosophie médicale de Sydenham ", Dialogue, IX (1970), p. 54-68 ; "Locke et le savoir de probabilité ", Dialogue, XI (1972), p. 185-203. Berkeley est entièrement gagné à la distinction d'une science rationnelle a priori de la nature, inaccessible à l'homme, et d'un savoir descriptif des phénomènes naturels. Mais, à notre avis, encore moins que chez Locke, on ne trouve 
réductible à la seule existence d'idées ; or aucune idée n'est active, puisque la seule nature de l'idée consiste à être perçue ; la causalité réelle ne peut donc appartenir qu'à un esprit dont la volonté est capable de faire apparaître les sensations dans mon esprit, ce qui n'est pas proprement en mon pouvoir, ni en celui d'aucun esprit analogue au mien quant à la limitation naturelle de sa volonté. Dieu est considéré par Berkeley comme l'auteur de ce langage des représentations naturelles qui exprime sa volonté ; et l'interprétation objective des données sensibles et de leur corrélation naturelle nous fait entrer dans les desseins de sa Providence. La seule vraie science est en Dieu : si l'homme veut construire un savoir rationnel a priori, il substitue sa volonté à celle de Dieu et n'aboutit qu'à un langage sans fondement ${ }^{12}$.

En fait, la position de Berkeley est sujette à des difficultés considérables qui proviennent d'une ambiguité dans sa doctrine de l'entendement, ambiguïté qui n'est certainement pas étrangère à l'exposé que Kant fait des paralogismes de la raison pure dans la Dialectique transcendantale. Sa critique de l'idée de matière repose sur le principe que le réel n'est intelligible que circonscrit dans les limites de la représentation: dans ces conditions, l'activité de la raison n'est que la consécution empirique des représentations ou idées ; mais cette consécution empirique ne devient intelligible que dans et pour un esprit, qui, lui-même, n'est pas réductible à une suite d'idées, puisqu'il est sujet percevant ${ }^{13}$.

dans sa philosophie le cadre épistémologique permettant d'établir les droits à la rationalité de ce savoir descriptif. Berkeley prétend que la science moderne incline à produire une cosmologie matérialiste et sceptique. C'est cette " métaphysique " de la science qu'il entend ruiner dans ses prétentions, mais, par le fait même, c'est avec une nouvelle doctrine métaphysique (celle-ci spiritualiste) qu'il entend concilier la science.

12. Berkeley, se servant de sa critique des substances matérielles supposées hors de l'esprit, conclut que les philosophes devraient admettre: 1) qu'il n'y a pas d'autre cause efficiente que quelque esprit : 2) que la considération des causes finales est légitime ; 3) que l'ordre de l'univers repose sur la volonté du Créateur ; 4) que la science de la nature se fonde sur des observations constantes et sur l'inférence de lois généralęs. Mais il ajoute dans les Principles, 107 : "I do not say "demonstrate ", for all deductions of that kind depend on a supposition that the Author of Nature always operates uniformly and in a constant observance of those rules we take for principles, which we cannot evidently know. "En effet, si l'ordre de la nature n'est qu'un langage, il n'y a pas de déductions a priori possibles concernant ce langage, à moins d'en être lauteur.

13. Nous croyons qu'il y a une difficulté majeure dans la notion d'idée, telle qu'elle est utilisée par Locke pour représenter tout ce qui se trouve dans l'esprit lorsqu'il 
Peut-être Berkeley rencontre-t-il alors les thèmes propres à l'analyse du cogito chez Malebranche, en particulier, que, comme objet, je ne puis avoir qu'une connaissance analogique et symbolique de la nature de ma propre "mens"; mais pour lui, l'entendement, comme pouvoir de signification ou de pensée, nous permet de connaître ce qui ne correspond à aucun donné sensible, en déterminant le caractère de la pensée comme activité. S'il affirme: "such is the nature of spirit, or that which acts, that it cannot be of itself perceived, but only by the effects which it produces ${ }^{14}$, c'est pour affirmer que nous connaissons néanmoins notre moi percevant. On peut toujours imaginer un rapport d'analogie entre des idées et le sujet percevant : ce rapport d'analogie renverrait alors à un autre rapport, celui-là non représentable, celui d'une causalité réelle propre à l'esprit, productrice de modification dans la consécution des idées ${ }^{15}$. Ce dernier rapport n'est compris et interprété que si l'entendemant n'est pas circonscrit dans les limites du représentable ${ }^{16}$. Berkeley utilise le terme de "notion" pour

pense, car, par le fait même, il tend à assimiler les concepts à l'état de simples représentations issues originellement de l'expérience des sens. D'où l'ambivalence du terme même de "représentation", qui, implique, d'une part, une correspondance à un objet visé par l'acte de l'esprit, et qui, d'autre part, désigne ce qui est directement présent à l'esprit, ce que l'on se représente (imagination), ce qui est le contenu concret, la " matière " d'un acte de pensée. Cette ambivalence se trouverait chez Descartes, comme chez Gassendi. Mais Locke et Berkeley se rattachent davantage à la tradition que représente celui-ci, lorsqu'il conteste l'existence d'une intuition proprement intellectuelle, pour réduire l'idée à la nature sensible de corrélat d'un acte d'imagination; toutefois Gassendi avait maintenu l'existence d'un entendement discursif qui, à partir de telles idées comme signes, poursuit l'intellection de ce qui est non représentable sous des espèces sensibles. C'est à ce type de conception qu'avec de notables variantes, se rattache l'usage des termes idée et représentation chez Locke et Berkeley, à cette différence près, que Berkeley réduit nettement l'idée à être le corrélat de la perception sensible, ce qui l'engage dans la voie d'une distinction entre connaissance des esprits et connaissance des idées, avec les apories épistémologiques que cette distinction peut impliquer.

14. Berkeley, Ibid., 27.

15. En fait Berkeley, distinguant perception et volonté comme actes du sujet percevant humain, affirme que la volonté n'agit pas à proprement parter pour produire les représentations ou idées (suis-je la cause de mes propres sensations?), mais que la volonté peut modifier l'acte de perception de façon à recomposer les rapports entre mes perceptions (d'où les idées qui relèvent de l'imagination et de la mémoire).

16. Il est indubitable que, dans les Principles, 142, Berkeley affirme la distinction ontologique et épistémologique des esprits et des idées: "Spirits and ideas are things so wholly different that when we say 'they exist', 'they are known', or the like, these words must not be thought to signify anything common to both natures." Mais, si la distinction est définie lorsqu'il s'agit de distinguer deux types d'être : 
représenter ce qui a une signification sans être réductible à des idées ${ }^{17}$. En définitive, ce n'est pas seulement l'esprit dont on a une notion sans avoir d'idée (cet esprit étant d'ailleurs soit le mien, soit celui des autres hommes, soit celui de Dieu), mais toutes formes de relation : "It is also to be remarked that, all relations including an act of the mind, we cannot so properly be said to have an idea, but rather a notion of the relations or habitudes between things ${ }^{: 8}$. D'où le problème posé par le pouvoir de comprendre et d'interpréter le langage naturel des représentations, problème tout entier contenu dans l'affirmation de Berkeley concernant la corrélation des phénomènes dans la formulation des lois physiques : " $\mathrm{A}$ man may well understand natural signs without knowing their analogy $" 19$. Cette affirmation illustre l'ambiguïté d'une doctrine de la raison qui tente de réduire l'intelligibilité du réel externe au contenu de la représentation. Comment puis-je reconnaître mes représentations comme signes, comme éléments du langage "naturel " de la Providence divine, si ce n'est par un acte de l'entendement qui transgresse les limites de la représentation ? ${ }^{20}$. Ce qui suppose que notre entendement, dans la relation de perception, a un fondement réel lui permettant de s'étendre au-delà du perceptible. Quel est ce fondement? Est-il pouvoir de Dieu ou pouvoir de la nature? Interrogation et dilemme qui se trouvent à l'origine des tentatives philosophiques pour déduire l'entendement. Il est indéniable que pour Berkeley, la voie empiriste de l'analyse des idées comme signes renvoyant aux éléments de la représentation sensible est la source d'apories irréductibles. Mais cette critique vient en

l'un, sujet, actif, percevant ; l'autre, objet, passif, perçu, elle implique un problème épistémologique lorsqu'il s'agit de connaître l'esprit, en tant qu'objet de connaissance. Ma notion de mon moi percevant n'est alors qu'hypothétiquement. distincte de toute relation à mes idées (ne serait-ce que la relation aux effets que l'esprit produit). La description psychologique, nécessairement inadéquate à l'être de la mens, n'en constitue pas moins un élément de mon expérience et de ma "représentation".

17. Cf. l'addition de 1734 au texte de 1710, Principles, $27:$ "Though it must be owned at the same time that we have some notion of soul, spirit, and the operations of the mind, such as willing, loving, hating - in as much as we know or understand the meaning of those words. " Remarquons que les mots étant des signes généraux, dans un cas comme celui-ci, le sens des termes généraux est indépendant des représentations particulières, ce qui laisse place à un certain conceptualisme.

18. Berkeley, Ibid., 142.

19. Berkeley, Ibid., 108.

20. Pour Berkeley, il s'agit d'une sorte d'illumination intérieure qui a son origine (sa cause et son sens) dans la volonté de Dieu. 
partie d'une position du problème suivant laquelle l'idée est radicalement détachée de la relation conçue par l'esprit, caractéristique de l'activité même de l'entendement mais inaccessible à la représentation, du moins suivant une détermination berkeleyenne. Une analyse empiriste attentive aux "relations " constitutives des idées dans le passage du simple au complexe et, par le fait même, attentive aux " relations $\bullet$ des idées aux signes du langage, pouvaitelle s'inscrire dans un dépassement des apories berkeleyennes? La question se trouve plus particulièrement à l'arrière-plan des discussions sur l'origine du langage chez Condillac. En résolvant ce nouveau problème, c'est, pense-t-on, le problème de la nature des idées et du langage des représentations qu'on se trouvera résoudre, sans recourir à l'explication métaphysique de l'ordre rationnel des idées par l'action du sujet percevant divin. Le point de départ de Condillac est l'analyse des instruments de connaissance chez Locke, en particulier son analyse du langage. D'un point de vue critique, un retour à Locke s'impose donc par-delà les analyses de Berkeley.

\section{II - L'ANALYSE LOCKIENNE DES SIGNES ET DES RELATIONS DE CONNAISSANCE}

L'analyse du langage dans l'Essay concerning Human Understanding répondait au désir de compléter l'étude des idées comme instruments et matériaux à l'aide desquels nous construisons le savoir, par une étude des mots comme signes des idées. Car les idées sont intimement reliées par l'esprit à des mots, et cette relation a lieu constamment dans le cas des mots généraux et des idées abstraites ${ }^{21}$. D'autre part, notre savoir porte principalement sur des propositions, en particulier sur les propositions les plus communément universelles, et il n'y a pas de propositions sans l'usage de signes généraux ${ }^{22}$. Enfin, il ne faut pas perdre de vue qu'en définissant la sémiotique comme l'une des trois grandes branches du savoir, Locke conçoit la doctrine des signes - ces signes sont aussi bien les mots que les idées - comme pouvant déboucher sur une nouvelle forme de logique et de critique, c'est-àdire sur une nouvelle forme de théorie du savoir et du jugement ${ }^{23}$.

21. Locke, Essay, II, xxxiii, 19

22. Locke, Ibid., III, i. 6.

23. Locke, Ihid., IV, xxi, 4. 
Deux problèmes dominent le livre III de l'Essay : 1) dans l'usage du langage, à quoi les mots sont-ils immédiatement appliqués? 2) puisque tous les noms, à l'exception peut-être des noms propres, sont généraux et représentent des espèces de choses, que sont ces espèces qu'ils représentent, comment se forment-elles dans la représentation? Chez les philosophes français d'allégeance condillacienne, ces deux problèmes deviendront : 1) comment le langage analyse-t-il la pensée? 2) quel est le principe qui régit la constitution des langues?

Positivement, pour Locke, les mots ne peuvent rien représenter d'autre que les idées de celui qui les choisit comme signes - choix arbitraire - afin de conserver ou de communiquer ses pensées. Mais nous référons en plus nos idées à la réalité des choses. Dans le cas des noms d'idées simples et d'idées complexes de modes, leurs archétypes n'existant et n'étant reconnus que dans le représentation, la relation n'est pas douteuse. Mais il n'en est pas de même pour les idées de substances, où l'archétype supposé est extérieur au champ de la représentation : "It is a perverting the use of words, and brings unavoidable obscurity and confusion into their signification, whenever we make them stand for anything but those ideas we have in our own minds $n^{24}$. Deux phénomènes tendent à nous voiler le véritable rapport des mots et des idées : 1) nous utilisons souvent les mots par l'effet de l'éducation, de l'habitude, de l'ignorance, sans considérer attentivement les idées correspondantes ; 2) l'usage intervient pour nous faire croire qu'il y a une connexion naturelle des mots avec certaines idées, alors que le vrai rapport est : " a perfect arbitrary imposition $"^{25}$. Ce sont ces deux phénomènes que Locke entend dénoncer dans son analyse des termes généraux, ce qui permet d'interpréter sous son vrai jour l'explication qu'il donne des idées abstraites.

Cette explication est en effet subordonnée à l'examen de la question : comment parvenons-nous à des termes généraux? La thèse proposée est la suivante : "words become general by being made the signs of general ideas : and ideas become general by separating from them the circumstances of time and place, and any other ideas that may determine them to this or that particular exis-

24. Locke, Ibid., III, ii. 5 .

25. Locke, Ibid., III, ii. 8. 
tence ${ }^{26}$. Autrement dit, l'abstraction serait cet acte de l'esprit par lequel il sépare d'une idée particulière toute représentation circonstancielle, pour en faire une idée générale. Mais, toute représentation étant nécessairement particulière, une telle idée générale n'est pas à proprement parler une représentation, et, sans doute, Berkeley prête-t-il trop facilement à Locke un point de vue génétique sommaire sur l'abstraction. En fait, Locke se propose surtout de donner une interprétation "épistémologique "des idées générales comme termes généraux dans le langage de la pensée. Il est clair pour lui que le général et l'universel n'appartiennent pas à l'existence réelle des choses, ni n'appartiennent par conséquent à l'existence des idées dans l'esprit : ce sont seulement, dit-il, "the inventions and creatures of the understanding, made by it for its own use, and concern only signs, whether words or ideas ${ }^{27}$. La signification de l'expression "idée générale " est donc à réviser, car il ne s'agit que d'une relation ajoutée à quelque idée particulière par l'esprit de l'homme. Toutefois le problème philosophique lié à cette interprétation ressurgit, lorsque l'on considère que les mots généraux signifient des espèces de choses.

En effet, quand on se demande quelle est la signification des mots généraux, on est amené à constater qu'ils ne signifient pas individuellement, chacun, une chose particulière, mais qu'ils signifient une pluralitée ${ }^{28}$, et qu'ils ont une fonction de classification par rapport aux objets de l'expérience. Or la signification de chaque espèce de choses est représentée par une essence. Puisque le nom, dans le cas des idées de substances particulières, ne correspond à aucun archétype connu par-delà les représentations qui constituent l'expérience du sujet connaissant, c'est donc que l'essence signifiée par un terme général correspond aux éléments de la représentation évoqués par le terme, et, par le fait même, l'idée abstraite devient coextensive à certaines images ou représentations particulières; s'ensuit-il que l'idée abstraite soit considérée comme un mode réel de l'esprit, alors que tout mode réel de l'esprit, en tant qu'on en a conscience, est nécessairement concret? Dans l'esprit de Locke, le fait que l'idée abstraite soit coextensive à des images n'implique

26. Locke, Ibid., III, iii, 6 ; c'est précisément cette formule prise comme définition de l'idée abstraite que Berkeley attaque dans l'introduction du Treatise concerning the Principles of Human Knowledge.

27. Locke, Ibid., III, iii, 11.

28. Locke, Ibid., III, iii, 12. 
pas que l'idée ne signifie davantage que le mode ou l'état actuel de l'esprit : c'est même pour éviter cette réduction que Locke s'attache à développer la relation épistémologique de l'idée par rapport aux données de l'expérience. Aussi pour échapper à la contradiction, - cette même contradiction que dénoncera Berkeley par la suite, - Locke établit sa notion d'essence nominale, par opposition à l'hypothèse d'essences réelles, mais inconnaissables, en vertu desquelles nous établirions la classification générique des éléments ou idées particulières de notre expérience. Il raisonne de la manière suivante : si les essences ne sont pas représentables, elles sont inconnaissables, et, comme telles, elles ne peuvent servir de structures pour la sélection et la classification des idées particulières ; il reste donc une seule possibilité de justifier la classification des phénomènes naturels: par référence à des structures de la représentation ayant un caractère général, structures qui deviennent intelligibles par l'acte d'abstraction de l'esprit sur le donné des idées particulières. "And what are the essences of those species set out and marked by names, but those abstract ideas in the mind; which are, as it were, the bonds between particular things that exist, and the names they are to be ranked under? " Cette thèse peut-elle être considérée comme une réponse anticipée aux objections de contradiction et d'absurdité que formule Berkeley?

La méthode descriptive que Locke utilise s'applique à l'observation des structures où sont impliquées les idées, mais Locke refuse de l'appliquer à l'examen de la nature même des idées, et surtout à la recherche de leur cause productrice ${ }^{30}$. Certes, pour répondre aux critiques de Norris et pour effectuer le réfutation de la théorie de Malebranche sur la vision en Dieu, il fournira une explication "métaphysique " des idées, qui se fonde sur l'extension de l'interprétation mécaniste des phénomènes naturels aux éléments mêmes de la représentation ${ }^{31}$. Mais cette explication n'a qu'une valeur de probabilité, et il lui semble préférable de s'en

29. Locke, Ibid., III, iii, 13.

30. Locke, Ibid., (éd. Fraser), Introd., 2 : " I shall not at present meddle with the physical consideration of the mind; or trouble myself to examine wherein its essence consists ; or by what motions of our spirits or alter ations of our bodies we come to have any sensation by our organs, or any ideas in our understanding, and whether those ideas do in their formation, any or all of them, depend on matter or not."

31. Cf. Remarks upon some of Mr. Norris' Books, sect. 17, The Works of Jobn Locke (10e éd.), 1801, tome X, p. 256 : "Ideas may be real beings, though not substan- 
tenir à l'ignorance professée dans l'Essay, où l'explication mécaniste elle-même est présentée comme reposant sur la distinction des idées de qualités premières et des idées de qualités secondes, distinction qui est en fait la distinction de deux langages, l'un plus général et plus fondamental que l'autre - le langage des qualités premières correspondant, semble-t-il, aux catégories mêmes du réel et de l'esprit. La véritable explication du mécanisme de l'abstraction se trouve plutôt dans la définition de la raison, non seulement considérée comme pouvoir de percevoir la connexion nécessaire des idées, mais aussi comme pouvoir d'étendre les idées suivant les indications de l'analogie $e^{32}$.

Mais qu'est-ce qui rend le rapport d'analogie significatif? En interprétant l'Essay, on en vient à formuler l'hypothèse qu'il s'agit d'une doctrine de la finalité naturelle, qui s'exprime à la fois dans l'ordre constant des phénomènes naturels, dans la constitution des habitudes de pensée et dans le pouvoir représentatif des catégories premières de la connaissance : idées simples de la sensation et de la réflexion. Mais, cette finalité naturelle étant irréductible à l'expérience, il faut se fier à la méthode historique pour fonder un savoir des réalités, ce qui explique que les idées, chez Locke, n'aient aucune rationalité a priori. Les idées deviennent des modèles, des types intelligibles, lorsque l'esprit leur donne une signification, mais elles restent alors toujours inadéquates à l'expression de la vraie logique qui régit la nature. C'est dans cette perspective que la sémiotique, comme critique des instruments de connaissance, prend tout son sens : elle permet la discrimination du savoir objectif et du savoir de pure institution. L'idée abstraite n'existe que comme attention de l"esprit à tel ou tel élément ou à tel ou tel groupe d'éléments de la représentation. Cette attention de l'esprit est un acte de réflexion qui implique la rationalité de l'esprit. Cette adéquation de la raison et de la réflexion, qui est aussi adéquation de la raison et de-l'ordre, constitue l'héritage cartésien, tel qu'il s'exprime dans l'Essay de Locke. Mais pour le philosophe anglais, si la réflexion

ces ; as motion is a real being, though not a substance ; and it seems probable that, in us, ideas depend on, and are some way or other the effect of motion; since they are so fleeting; it being, as I have elsewhere observed, so hard, and almost impossible, to keep in our minds the same unvaried idea, long together, unless when the object that produces it is present to the senses; from which the same motion that first produced it being continued the idea itself may continue. "

32. Locke, Essay, II, xvii, 2. 
de l'esprit sur les éléments de la représentation aboutit à la conception d'idées-essences, le savoir que l'imagination produit à partir de ces essences abstraites n'est que nominal : il n'est confirmé ou infirmé que par les effets de nos habitudes de pensée dans la pratique de l'existence. De même qu'il rejette l'hypothèse cartésienne du rêve continu ${ }^{33}$, Locke rejette la doctrine d'un savoir certain des réalités naturelles dans leur " essence "; il n'y a plus de certitudes absolues, seulement des certitudes suffisantes pour l'existence humaine. Il assigne à l'homme de science, comme seule tâche raisonnable, la description de la nature. Il définit ainsi une position philosophique qui sera l'une des caractéristiques des philosophes français au XVIIIe siècle, caractéristique que l'on retrouve par exemple dans les Éléments de philosophie (1759) de d'Alembert. En fin de compte, l'analyse lockienne contourne le problème soulevé par Berkeley en restreignant l'ampleur de la déduction de l'entendement à la dérivation empirique des idées qui servent à constituer la description rationnelle des réalités, par comparaison avec celles qui entrent dans la formation des connaissances issues de la combinaison, relation et analyse des notions complexes de mode.

C'est dans la mesure où Locke propose une description des instruments du savoir et de leur usage légitime, plutôt qu'une déduction de l'entendement humain, que sa doctrine des idées abstraites échappe aux analyses critiques de Berkeley. Mais, dans un autre registre d'analyse, les attaques de Berkeley contre l'existence réelle des idées abstraites, en tant qu'éléments de la représentation, semblent néanmoins difficilement évitables aux successeurs de Locke. Tout en conservant l'enseignement lockien sur la méthode, la possibilité de constitution et les limites d'un savoir portant sur l'expérience des réalités naturelles, ils devront envisager de le corriger sur un point fondamental: il leur faudra tenter de tirer la raison de la nature empirique de l'homme et de déduire son pouvoir de réflexion des éléments mêmes de la représentation. Rien de plus significatif à cet égard que le dessein de Condillac dans l'Essai sur l'origine des connaissances bumaines (1746).

33. Locke, Ibid., IV, ii. 14. 


\section{III - CONDILLAC ET LA DÉRIVATION EMPIRIQUE GÉNÉRALISÉE}

Condillac affirme: "nous ne devons aspirer qu'à découvrir une première expérience que personne ne puisse révoquer en doute et qui suffise pour expliquer toutes les autres. Elle doit montrer sensiblement quelle est la source de nos connaissances, quels en sont les matériaux, par quel principe ils sont mis en ceuvre, quels instrumens on y emploie et quelle est la manière dont il faut s'en servir. ${ }^{34}$ Et il reproche à Locke de n'avoir pas vu que, dans l'étude de l'entendement, c'est la question de l'origine des facultés qu'il fallait examiner : il n'est pas suffisant d'affirmer que l'âme reçoit toutes ses idées des sens, et que l'esprit possède le pouvoir de les répéter, de les composer, de les unir et de faire toutes sortes de notions complexes; il fallait " expliquer la génération des opérations de l'âme ${ }^{35}$.

L'analyse génétique de Condillac et la théorie de la sensation transformée sont assez connues pour que nous nous dispensions de les rappeler ici. Retenons seulement le principe d'ensemble de l'analyse et son fondement philosophique. Condillac fait des sensations et des opérations de l'âme - celles-ci réduites complètement à celles-là dans le Traité des Sensations (1754), par suite de l'analogie qui les lie - les matériaux de toutes nos connaissances, matériaux que la réflexion, qui n'est que l'analogie naturelle devenue conscience, met en œuvre en opérant l'analyse des rapports qui y sont impliqués. Le fondement philosophique de l'analyse est fourni par des considérations subtiles pour évincer le problème métaphysique de l'hétérogénéité de l'âme et du corps : Condillac développe une théorie occasionnaliste qui, en même temps, subordonne toute connaissance au fait que dans l'état actuel — postérieur au péché originel - nous n'avons point d'idées qui ne nous viennent des sens ${ }^{36}$. Ce sera le point de départ des analyses du Traité des Sensations, où il entend distinguer sa philosophie de celle de l'idéa-

34. Condillac, Essai sur l'origine des connaissances bumaines, introduction, Fuvres philosophiques de Condillac, Paris, P.U.F., 1947, tome I, p. 4A (nous désignerons par l'abréviation O.P. cette édition des ceuvres de Condillac).

35. Condillac, Ibid, introduction, O.P., tome I, p. 5B.

36. Condillac, Ibid., II, I, i., 8, O.P., tome I, p. 7B : "L'âme étant distincte et différente du corps, celui-ci ne peut être que cause occasionnelle de ce qu'il paroît produire en elle.." 
liste Berkeley: si la sensation est modification de l'âme, avec l'expérience du toucher dans le mouvement, la sensation devient celle d'un double contact, ou d'une résistance qui produit la conscience de l'extériorité de l'objet. Remarquons-le: c'est de l'intérieur de l'expérience sensible, à partir des données mêmes de la sensation, que se produit cette prise de conscience ${ }^{37}$. Mais peut-on inférer de l'expérience sensible la genèse des abstractions, c'est-àdire des représentations-signes ayant une signification générale?

L'usage des signes, explique Condillac, dépend de l'imagination qui réveille une perception par l'effet de la liaison que l'attention a établie entre cette perception et un objet, lorsque celui-ci se présente de nouveau à l'expérience sensible; il dépend aussi de la mémoire qui, à défaut de la perception même, en rappelle les circonstances ou signes, le nom par exemple, mais ces signes ne sont en fait que des perceptions concomitantes à la perception initiale, qu'il est impossible ou inutile de se remémorer ; la réminiscence, enfin, identifie le signe en faisant connaitre la relation qu'il a aux perceptions qu'on a déjà eues ${ }^{38}$. Mais la possibilité de ce déplacement de l'attention, qui permet l'usage de certaines perceptions comme signes, repose sur une liaison des idées suivant une sorte de logique ou d'analogie naturelle, qui est ainsi exprimée: "La liaison de plusieurs idées ne peut avoir d'autre cause que l'attention que nous leur avons donnée quand elles se sont présentées ensemble: ainsi les choses n'attirant notre attention que par le rapport qu'elles ont à notre tempérament, à nos passions, à notre état, ou, pour tout dire en un mot, à nos besoins ; c'est une conséquence que la même attention embrasse tout-à-la fois les idées des besoins et celles des choses qui s'y rapportent, et qu'elle les lie. $n^{39}$

Les besoins fourniraient une suite d'idées fondamentales auxquelles se rattacheraient toutes nos perceptions. Condillac reprend l'une des idées maîtresses des Regulce de Descartes, celle d'une unité réelle des déductions possibles à partir des diverses notions premières, mais il transforme la chaîne des évidences, comme trame des raisons pour l'esprit et comme expression de l'ordre naturel, en une suite d'idées " dont la force serait entièrement dans l'analogie des signes, dans l'ordre des perceptions et dans la liaison

37. Condillac, Traité des Sensations, II, v, 1, 1-2, O.P., tome I, p. 243-255.

38. Condillac, Essai, 1, II, ii, 25, O.P., tome I, p. 16B.

39. Condillac, Ihid., I, II, iii, 28, O.P., tome I, p. 17A-B. 
que les circonstances qui réunissent quelquefois les idées les plus disparates auraient formée ${ }^{40}$. La raison serait-elle alors une sorte d'instinct supérieur? Condillac fait remarquer que si l'esprit de l'homme était limité aux signes accidentels et naturels que l'expérience lui révèle, il n'aurait pas de pouvoir à proprement parler sur de tels signes, sa mémoire serait réductible à l'imagination, et il se comporterait comme les bêtes, dont l'instinct n'est qu'une imagination capable de les diriger sans l'aide de la réflexion. L'homme s'émancipe des limitations naturelles de l'esprit, lorsqu'il est capable de rattacher ses idées à des signes artificiels - acte qui implique une détermination consciente - ce qui lui permet de contrôler et d'amplifier le cours de son imagination ${ }^{41}$. Le problème de la raison humaine devient celui de l'invention des signes artificiels.

Condillac est en effet capable de tourner la difficulté que Berkeley rencontrait dans la doctrine des idées abstraites de Locke, mais, par le fait même, il reporte la difficulté sur la question de l'origine du langage. Les idées abstraites ne sont plus considérées que comme "des dénominations que nous donnons aux choses envisagées par les endroits par où elles se ressemblent ; c'est pourquoi on les appelle idées générales ${ }^{42}$. Elles se forment lorsque l'attention se porte vers les propriétés des choses par où elles conviennent, en se détournant des qualités par où elles se distinguent ${ }^{43}$. Les perceptions " génériques" ainsi acquises ne sont pas proprement des signes, mais il suffit que l'esprit fixe son attention sur l'analogie possible de ces perceptions avec des mots par exemple, pour accéder à des notions générales. Cette analogie ne peut être saisie que si l'esprit a déjà acquis le pouvoir d'étendre ses perceptions par une mémoire élémentaire ${ }^{44}$. Ce qui explique peut-être l'un des pa-

40. Condillac, Ibid., I, II, iii, 29, O.P., tome I, p. $17 \mathrm{~B}$.

41. Condillac, Ibid., I, II, iv, 46, O.P., tome I, p. 21B : " Dans la suite, il acquerra d'autant plus d'empire sur son imagination, qu'il inventera davantage de signes, parce qu'il se procurera un plus grand nombre de moyens pour l'exercer."

42. Condillac, Ibid., I, V, 1, O.P., tome I, p. 49A.

43. Condillac, Ibid., I, II, vi, 57, O.P., tome I, p. 24A ; et Traité des Sensations, IV, vi, 4-6, O.P., tome I, p. 306-307. Dans ce dernier texte, Condillac annonce très nettement la doctrine des images génériques, lorsqu'il affirme (sec. 6, O.P., I, p. 307B) : "Elle [la statue] généralise donc davantage, à proportion qu'elle voit d'une manière plus confuse ; et elle se fait des notions moins générales, à propor. tion qu'elle démêle plus de différence dans les choses."

44. C'est ce que Condillac exprime lorsqu'il dit dans le Traité des Sensations, IV, vi, 4 (addition postérieure à 1754), O.P., tome I, p. 307A : "Comme la statue n'a 
radoxes de la "noogénie " de Condillac. D'une part, il affirme en effet que le commerce réciproque des hommes est indispensable pour que les perceptions deviennent objets de réflexion : auparavant " elles ne sont que des impressions faites dans l'âme, auxquelles il manque pour être des idées d'être considérées comme image "45. D'autre part, il lie ce commerce à des actes de connaissance qui ne sont que des "sensations transformées". Le paradoxe s'estompe, lorsque, par les opérations du toucher dans le mouvement, la statue a pris conscience de l'existence des corps : car l'expérience des corps est la condition première et l'instrument de la communication des hommes par le langage ${ }^{46}$. Le véritable problème de l'abstraction est alors d'expliquer l'analogie qui lie le langage et la raison comme pouvoir de combiner des idées par des compositions et des décompositions qui fassent ressortir de nouvelles relations.

On connaît l'affirmation célèbre de Condillac dans la Langue des Calculs : "Toute langue est une méthode analitique, et toute méthode analitique est une langue. ${ }^{47}$ Comment est-il parvenu à l'énoncé d'une proposition aussi ambiguë ? D'une part, Condillac interprète la pensée logique comme soumise à la loi de l'enchainement analogique des perceptions et des signes, qu'il a mise en évidence dans l'Essai sur l'origine des connaissances et que nous avons déjà indiquée ${ }^{48}$. Par ailleurs, il donne une analyse de l'origine des langues qui est destinée à mettre en valeur l'empire souverain de l'association analogique jusque sur les éléments "physiques " du pouvoir de représentation, c'est-à-dire sur les conditions matérielles de la pensée réfléchie. Il utilise ainsi la notion de "langage d'action " pour qualifier les gestes, les mouvements du visage

l'usage d'aucun signe, elle ne peut pas classer ses idées avec ordre, ni par conséquent, en avoir d'aussi générales que nous. Mais elle ne peut pas non plus n'avoir absolument point d'idées générales. Si un enfant qui ne parle pas encore, n'en avoit pas d'assez générales pour être communes au moins à deux ou trois individus, on ne pourroit jamais lui apprendre à parler, car on ne peut commencer à parler une langue, que parce qu'avant de la parler, on a quelque chose à dire, que parce qu'on a des idées générales: toute proposition en renferme nécessairement. "

45. Condillac, Essai, I, IV, ii, 25, O.P., tome I, p. 47B ; ce point de vue semble confirmé par le Traité des Sensations, II, viii, 35, O.P., tome I, p. 267B-268A.

46. Condillac semble à cet égard annoncer la triple corrélation de l'étendue, du langage et de l'intelligence chez Bergson.

47. Condillac, Langue des Calculs, objet de cet ouvrage, O.P., tome II, p. 419A,

48. Cf. De l'art de Penser, I, v; tout le début du chapitre est une reprise de l'Essai, I, II, iii (O.P. tome I, p. 726 et p. 17-19). 
et les sons inarticulés qui sont comme l'expression naturelle de nos besoins, dans la mesure où ils permettent à l'esprit de signifier une perception. Condillac forge un modèle théorique avec la supposition de deux enfants égarés dans quelque désert avant de connaître l'usage d'aucun signe. "Quand ils vécurent ensemble, affirme-t-il, ils eurent occasion de donner plus d'exercice à ces premières opérations [grosso modo, il s'agit des opérations dont la statue dans le Traité des Sensations sera, par la suite, capable], parce que leur commerce réciproque leur fit attacher aux cris de chaque passion les perceptions dont ils étaient les signes naturels ${ }^{49}$. Ce langage d'action est celui des besoins naturels qu'il exprime en fonction de la conformation physique de l'espèce. Par l'habitude de rattacher ainsi des perceptions à l'expression sensible du corps, la mémoire des utilisateurs du langage d'action se développa considérablement; puis ils en vinrent à lier quelques perceptions à des signes arbitraires, - au départ simples effets des contingences naturelles, - ce qui leur donna occasion de faire un nouveau langage suivant le modèle des cris naturels. Et encore le sens des premiers mots qu'ils créèrent dut-il être ajusté grâce aux signes du langage naturel, qui fournissait des circonstances, ou perceptions remémorées, ou signes concomitants, que chacun pouvait rapporter aux mêmes perceptions ${ }^{50}$. Voilà pourquoi, dans sa Grammaire, Condillac refuse d'appeler "signes arbitraires" les mots de nos langues, qui ne sont, à son avis, que des «signes artificiels" choisis en raison, c'est-à-dire suivant l'analogie naturelle des perceptions et pour les représenter ${ }^{51}$. En entrant dans le détail des analyses de Condillac sur le langage, on reconnaît sans difficulté que, pour lui, la raison est le pouvoir d'étendre nos perceptions par. l'usage des signes : cet usage exprime des rapports d'analogie. Or "l'analogie est proprement un rapport de ressemblance " et " une chose peut

49. Condillac, Essai, II, I, 1, 2, O.P., tome I, p. 60B-61A ; développements très voisins sur le langage d'action comme origine du langage des sons articulés dans la Grammaire, I, i, O.P., tome I, p. 428-431.

50. Condillac, Essai, II, I, ix, 80, O.P., tome I, p. 83A : "Le langage d'action levoit les ambiguités et les équivoques qui, dans les commencemens, devoient être fréquentes."

51. Condillac, Grammaire, I, i, O.P., tome I, p. 429A : "En effet, qu'est-ce que des signes arbitraires? Des signes choisis sans raison et par caprice. Ils ne seraient donc pas entendus. Au contraire des signes artificiels sont des signes dont le choix est fondé en raison : its doivent être imaginés avec tel art, que l'intelligence en soit préparée par les signes qui sont connus." 
être exprimée de bien des manières, puisqu'il n'y en a point qui ne ressemble à beaucoup d'autres ${ }^{32}$. Il s'ensuit deux principes d'in. terprétation de la raison humaine : 1) la raison est elle-même dans un rapport d'analogie par rapport à la nature : par conséquent, le développement matériel de l'humanité et également le développement physique de l'individu humain expriment, dans leur diversité, l'ordre de la raison; 2) l'invention rationnelle, pour l'homme, est conforme à l'analogie des opérations mêmes de la nature: en particulier, l'abstraction n'est qu'une analyse de la représentation par la représentation, suivant un ordre que la nature indique elle-même : "c'est celui dans lequel elle offre [les] objets. Il y en a qui appellent plus particulièrement les regards; ils sont plus frappants ; ils dominent ; et tous les autres semblent s'arranger autour d'eux pour eux ... On commence donc par les objets principaux : on les observe successivement, et on les compare pour juger des rapports où ils sont. Quand, par ce moyen, on a leur situation respective, on observe successivement tous ceux qui remplissent les intervalles, on les compare chacun avec l'objet principal le plus prochain, et on en détermine la position. Alors on démêle tous les objets dont on a saisi la forme et la situation, et on les embrasse d'un seul regard. L'ordre qui est entre eux dans notre esprit n'est donc plus successif. C'est celui-là même dans lequel ils existent, et nous les voyons tous à-la-fois d'une manière distincte. "s3 $^{33}$ L'idée abstraite n'est donc qu'une représentation-signe dont l'esprit se sert pour analyser sa pensée suivant le mode de la succession temporelle, mais l'essence correspondant à l'idée abstraite ne peut être que la totalité de la représentation dans son ordre. L'article Langue de l'Encyclopédie traduit à merveille cette pensée de Condillac : "L'esprit, y est-il écrit, vient à bout de distinguer des parties dans sa pensée, toute indivisible qu'elle est, en séparant, par le secours de l'abstraction, les différentes idées qui en constituent l'objet, et les diverses relations qu'elles ont entre elles à cause du rapport qu'elles ont toutes à la pensée indivisible dans laquelle on les envisage. $"{ }^{54}$

52. Condillac, Langue des Calculs, objet de cet ouvrage, O.P., tome II, p. 419A.

53. Condillac, Logique, I, ii, Que l'analyse est l'unique métbode pour acquérir des connatsances. Comment nous l'apprenons de la nature même, O.P., tome II, p. 375A-B

54. Encyclopédie, art. Langue, tome IX, p. 257A 
Ainsi se trouve achevée l'analyse critique des idées abstraites dans leur fonction représentative, analyse commencée avec Locke dans la perspective d'une sémiotique qui détache l'idée abstraite de son adéquation à une essence réelle supposée, pour ne plus la rapporter qu'au contenu même de la représentation. Mais Berkeley montrait, tout aussi bien, qu'il n'y a pas de représentation abstraite correspondant à l'acte même de perception de l'esprit : ce qui l'amenait alors à concevoir la rationalité du réel comme objet de postulation sous forme de notions par-delà toute possibilité de représentation; l'existence même de l'objet comme réalité indépendante du sujet percevant était niée ; les perceptions devenaient les signes de la volonté divine, mais l'interprétation de ce langage reposait sur la connaissance de "notions" qui se situaient hors du champ de la représentation possible. Condillac, reprenant Locke, n'entend point rapporter les éléments de la connaissance à une signification transcendante à l'esprit humain : il tente la genèse des opérations mêmes de l'âme, à partir d'un principe qui doit être fourni par l'expérience. Ce principe unique est la liaison analogique des idées qui est illustrée par l'usage des signes. Par le fait même, il est amené à tenter d'établir que la raison est le rapport analogique de la nature dans la représentation. Épistémologiquement, le champ est libre pour que s'élabore le projet de l'Encyclopédie. Sans doute, définit-il, dans la pensée du XVIIle siècle une troisième voie, qui n'est ni celle de Hume, ni celle de Kant. C'est cette voie que suivront les Encyclopédistes, en particulier lorsqu'ils concevront de rattacher l'analyse de l'état des connaissances scientifiques sur la nature à la structure d'une "idéogenèse " néolockienne. Indiquons seulement que la signification de l'analyse de Condillac se trouve profondément altérée dès le début du XIXe siècle, lorsque les sensations cessent d'être considérées comme de pures modifications de l'âme pour devenir l'expression du dynamisme du corps, comme cela apparaît, par exemple, à travers les Rapports du Physique et du Moral (1802) de Cabanis. Mais Condillac lui-même n'est-il pas responsable de cette transformation dans la mesure où il projetait de tirer de l'expérience le principe générateur des opérations de l'âme? Quant à l'analyse des idées abstraites, dans une telle orientation de la recherche, elle était appelée à devenir de plus en plus dépendante d'une psychologie de l'adaptation vitale.

Université d'Ottawa 